\title{
Securing Industrial Control Systems through Autonomous Hardening
}

\author{
Robin Chapas \\ ABB Corporate Research \\ robin.chapas@ch.abb.com
}

\author{
Ana Hristova \\ ABB Corporate Research \\ ana.hristova@ch.abb.com
}

Sebastian Obermeier

ABB Corporate Research

sebastian.obermeier@ch.abb.com

\author{
Thomas Locher \\ ABB Corporate Research \\ thomas.locher@ch.abb.com
}

\begin{abstract}
The secure and reliable operation of industrial control systems is becoming more and more challenging due to the increasing size, complexity, and heterogeneity of such systems. A constant change of requirements and responsibilities results in an increased frequency of configuration and topological changes, which renders a manual verification of system security infeasible. Thus, there is a need for automatic mechanisms that allows a system to uphold a desired level of security autonomously.

In this paper, we present a framework that enables a system to harden itself periodically, i.e., the framework ensures that each device complies to a security baseline tailored to the device's functionality and capability. The evaluation of our implementation shows that the framework effectively and efficiently corrects any deviations from the desired state at each networked device and thereby guarantees that the overall system remains compliant to pre-defined security policies. Moreover, the scalability tests conducted in a cloud infrastructure indicate that the framework is suitable for fairly large networks, with hundreds of individual devices, which makes it suitable for a wide range of practical control systems.
\end{abstract}

Keywords: cyber security, security hardening, security automation

\section{INTRODUCTION}

Today's industrial control systems, which are responsible for the monitoring and control of, e.g., power grids, emergency communication systems, or industrial production processes, are becoming increasingly complex. As a result of the growing complexity and size, the management and control of such systems is continuously becoming a harder and more time-consuming task. However, a lack of constant supervision and adaptation to configuration changes can have a serious impact on the system's dependability and security. While a security breach in a corporate network may incur a substantial financial loss, the situation is more severe in industrial control networks, where an incident may lead to an outage of electric power, the contamination of potable water, or the disruption of a sewage disposal process, which can directly impact people's safety and health. It is evident that the security of such ICSs is of utmost importance.

Apart from installing dedicated security devices and ensuring a secure network topology, a crucial step to enhance system security is to harden all devices that are part of the system. Hardening is the process of securing a system by reducing its surface of vulnerability, e.g., by fixing software bugs that may be exploited, shutting down unutilized services, and running up-to-date anti-virus software and a welltuned firewall.

Hardening large systems is a challenging task not only because the systems are growing, but also because the proliferation of networked devices has caused a substantial increase in the rate of network changes, which further exacerbates the management and control problem. An additional problem in practice is the heterogeneity of the devices in the system: the devices have different functions and are positioned in different parts of the network, and therefore have different security requirements. Moreover, the devices may run various operating systems with different security capabilities on diverse hardware with different (spare) capacities for additional security control mechanisms. Finally, frequent changes in the configuration necessitate a periodic validation of the security settings at all devices. Since the time interval between validations may be small, especially in industrial control applications, an automated solution is required. Thus, there is a need to harden the entire system, i.e., all devices according to their 
individual functions and capabilities, continuously and autonomously to ensure a high level of security.

Given a set of potentially changing security policies, we present a mechanism that allows a heterogeneous system to harden itself periodically. Defining and maintaining the security policies themselves is a separate, challenging problem that is out of scope of this work. The contributions of this paper are the description of the proposed framework of an autonomous, scalable, and robust hardening system, a discussion of different implementation options, and also a thorough evaluation of a full implementation capable of continuously hardening systems with hundreds of individual devices. The implementation of our framework is evaluated with respect to various metrics such as bandwidth and CPU consumption, and the time required to harden all devices. The scalability of the implementation is analyzed as well by emulating hundreds of devices in Amazon's Elastic Computing Cloud ${ }^{1}$.

\section{RELATED WORK}

Mechanisms to protect and secure large networks have been extensively studied for several decades. Due to the increasing complexity of networked systems, there is a growing interest in self-managing systems capable of adjusting configuration parameters themselves so that the desired quality of service is guaranteed continuously. An autonomous, dynamic (re-)configuration requires advanced feedback control mechanisms to monitor the system state and crucial system metrics, and also to take appropriate actions. One approach is to use controllers that periodically try to determine the best configuration for the system by combining analytic performance models with combinatorial search techniques (Menascé and Bennani 2003). If the desired state of the network entities can be described using policies, they can be monitored and configured using NESTOR, an architecture for network selfmanagement and organization (Yemini et al. 2000). In this approach, policy scripts are used to access and manipulate the network entities via a resource directory server (RDS). CFEngine is a popular open source configuration management system whose goal is to manage and maintain the configuration of large computer networks. It uses a client-server architecture with agents running at the managed machines. Instead of specifying the steps that need to be carried out, the CFEngine language describes the desired state, and the agents must ensure that the clients converge to a compliant state (Burgess 1998). Another general system and service configuration framework is SmartFrog (Goldsack et al. 2003), which is responsible for service configuration, description, deployment, and life-cycle management. However, it does not support dynamic configuration

\footnotetext{
${ }^{1}$ See http://aws.amazon.com/ec2
}

at runtime. There are also several commercial system management products, e.g., IBM's Tivoli (Karjoth 2003), Microsoft's Security Compliance Manager or Triumfant's Security Configuration Management, which are typically centralized and agentbased as well.

A different approach was proposed specifically for database systems where each security configuration parameter change attempt or request is compared against an embedded predefined security policy (Jabbour and Menasee 2008).

There is also a significant amount of work on the autonomous, distributed detection and handling of security events. The Distributed Intrusion Detection System (Mukherjee et al. 1994) uses a centralized director that aggregates information obtained from monitors to detect intrusions. The monitors are installed in dedicated machines in the network. Several proposed architectures incorporate lightweight, independent agents working together to detect anomalous behavior, e.g., (Balasubramaniyan et al. 1998; Helmer et al. 1998; Spafford and Zamboni 2000). The NADIR (Hochberg et al. 1993) system collects audit data distributively, which is then analyzed by a central expert system. The EMERALD project (Porras and Neumann 1997) uses socalled service monitors at the hosts to monitor their operation. Various layers of monitors are defined to reduce the required data in a hierarchical manner. Lastly, the biological immune system has also been emulated to achieve an automated reaction to intrusion attempts (Forrest et al. 1997).

Our framework shares the basic structure of these distributed systems in that information is collected at a small number of central entities. However, the goal of our framework is neither to detect attacks nor to improve the ability of the system to detect or react to attacks; instead it strives to minimize the attack surface by ensuring that the system always adheres to the latest security policies. To the best of our knowledge, our framework is the first to present a unified solution to the challenging problem of continuously and autonomously hardening networked systems comprising vastly different system entities.

\section{A SYSTEM FOR AUTONOMOUS HARDENING}

After a short presentation of basic concepts and definitions, we introduce our framework, describe all the basic hardening techniques, and also discuss our implementation, including a summary of the advantages and disadvantages of various implementation options.

\subsection{Model \& Definitions}

In the context of industrial control systems, a system is modeled as a set of heterogeneous devices, 
such as intelligent electronic devices, remote terminal units, data historians, input/output servers, human-machine interfaces, and control servers, interconnected over a network. These devices have different physical and virtual characteristics and capabilities as well as different functionality, and are running different software on various operating systems. As discussed in the preceding sections, it is essential to decrease the available attack vectors of the system's components in order to increase the security level of the whole system. This can be accomplished through security hardening. Security hardening is defined as minimizing the attack surface by removing or disabling unnecessary software, user accounts, and services, closing unused ports, securely configuring available services etc.

Devices in the system that need to be hardened are called target machines or simply targets. As the targets are heterogeneous in nature, running on different hardware and software, they may have different security requirements. In our framework, we express a security requirement with the notion of a security setting. A security setting describes a certain security feature, e.g., "firewall activated", "port 23 closed", "guest user account disabled" etc. Every setting is associated with a value indicating the desired status. In the given examples, a Boolean value could be used to indicate that having an activated firewall is the desired state, i.e., true would be the desired value for this setting. Each target is associated with a security policy, which is a set of security settings. If there are targets with identical security requirements, they are associated with the same policy, i.e., it is not necessary to have a distinct policy for each target.

The goal of the framework, described below, is to provide a mechanism ensuring that current security policies are preserved in the system especially when the devices and their configurations change frequently. The framework allows the system to harden itself autonomously in that no operator intervention is required when some targets violate the security policy, either after initialization or due to configuration changes.

\subsection{Framework}

In order to perform security hardening in a heterogeneous distributed environment in a flexible and autonomous manner, a framework that is able to associate security settings with individual devices or device types is needed. As mentioned before, we assume that the desired security policies are available, i.e., an external mechanism is required that provides the framework with up-to-date security policies. Such a framework must further be able to audit and enforce the settings on all devices, which implies that it must know how to to retrieve the current values from all devices and compare the retrieved results to the desired values, and, finally, how to modify the settings. In short, the framework abstracts away from the heterogeneity of the devices and allows the user to specify the security policies in a device-independent manner. Moreover, the framework should offer multiple ways to address the devices, which enables the user to choose the best method depending on the target types.

\subsubsection{Alpha Machines}

In the following, we describe our approach to address these objectives. The framework consists of two parts: The network containing all target machines and the alpha machine cluster. The alpha machine cluster is a well-protected and trusted cluster of so-called alpha machines. The task of an alpha machine is to monitor the targets, communicate with them periodically, and correct deviations from the desired state by enforcing the desired settings. In order to ensure a high reliability of the system and the autonomous hardening mechanism, it is essential to have multiple alpha machines. An alpha machine that is monitoring the targets is called active, and any alpha machine that is not monitoring a target is called passive. There may be any number of passive alpha machines but there is at least one active alpha machine at any time. While there can be multiple active alpha machines splitting the workload of auditing and hardening the targets, the easiest set-up is to use one active alpha machine whose status is monitored by the passive alpha machines. A so-called view change occurs when an active alpha machine fails, where it is replaced automatically by running a distributed consensus algorithm such as Paxos (Lamport 1998) among the passive alpha machines.

Since the alpha machines have administrator rights on all targets, which are needed to retrieve and modify the security settings, they must be placed in a trusted network. All communication with targets runs over encrypted, authenticated channels. If it is not possible to segregate the alpha machine cluster from the rest of the network, additional security measures must be in place, e.g., the communication between the alpha machines must also be secured.

\subsubsection{Options \& Baselines}

As mentioned before, an active alpha machine periodically audits each assigned target and checks its local security settings. For this purpose, the alpha machine uses so-called options. An option defines how an individual security setting is audited and how a particular setting is enforced by specifying the commands that need to be executed at the target. Thus, each option consist of the following:

- A command to retrieve a specific security setting.

- A method to parse the output from the target.

- A method to compare it to a value in the security policy.

- A command to enforce a security setting. 
An ordered collection of options designed for a specific device or operating system type, together with a specification of the communication method used to interact with the target, is called a baseline. A baseline allows the alpha machine to audit and enforce the security policy at a certain target type. Different communication methods are discussed shortly. Multiple baselines for the same security policy may exist if there are distinct target types, e.g., machines running different operating systems but with the same security requirements. The framework allows an administrator to create such baselines, on any alpha machine, and associate them with the targets in the system. Hence, the mapping from the abstract policy description to the commands for the individual targets occurs at the alpha machines where the baselines are stored. Once a new baseline is added to an alpha machine, it is automatically disseminated among all alpha machines. The distribution of baselines must also be secured by authenticating and encrypting the communication channels if the alpha machines are not in a closed network. If the policies must be changed or updated, an administrator uses the same mechanism as when creating a new baseline. Since the policies typically do not change substantially, this manual step is not a significant limitation in practice; moreover, an external automated update mechanism can easily be added to the framework if there are frequent changes.

\subsubsection{Auditing \& Hardening}

An alpha machine can perform two main actions to secure the system: auditing and enforcing certain security settings at each target. The auditing and hardening process can be either interconnected or performed independently, i.e., the framework can also directly enforce certain settings without a preceding auditing phase. The distinction and separation of these two processes has several advantages: a) it enables a better reporting of discrepancies, $b$ ) it is possible to obtain an overview of critical settings that cannot be enforced easily or automatically, ${ }^{2}$ and c) it allows an operator to correct a deviation by selecting a value from a range of recommended values instead of applying one admissible value automatically.

As the alpha machines steer the auditing and hardening, a key aspect is how they interact with the targets. As discussed in Section 2, a classic approach is to communicate with agents installed at the target machines. An agent is a (light-weight) software component with dedicated functionalities. In our case, an agent is capable of receiving a list of auditing or enforcing commands, transmitted in XML format, which the agent executes on the target. The agent validates the information sent from the

\footnotetext{
${ }^{2}$ Particularly in industrial control systems, it is often not permitted to change certain settings in an automated manner. In this case, an operator must examine the identified discrepancies and perform changes manually.
}

alpha machine and it only executes correct auditing and enforcing commands. An agent communicates with an alpha machine through a dedicated port. In order to ensure secure communication, all data exchanged is encrypted and the authenticity of the communicating entities is verified. A suitable protocol for this purpose is SSL, which we have used in our implementation. The mutual authentication requires digital certificates at all targets and at the alpha machines.

This agent-based approach has both advantages and disadvantages. The main advantage of using an agent is that it is a dedicated piece of software developed and optimized for a specific purpose. It can implement the needed functionality and can apply the previously received settings as defined in the baseline periodically even when there is no connectivity to the alpha machine or no network connectivity at all. In addition, one can choose the encryption mechanism and can define the priority of this process at the target machine. On the other hand, the main drawback of this approach is that it is rather intrusive and only useful for the designed functionality. For some critical network components, it may not be allowed to have agents installed because the component may otherwise not be able to meet certain performance requirements.

Therefore, it is necessary to support alternative approaches to perform the auditing and hardening processes. If there is no agent, then the target itself must have some means of accepting and executing commands. There are various remote code execution methods for this purpose such as PsExec, SSH, Windows PowerShell, RemCom, Rexec, Remote Shell (RSH)/REMSH/RCMD, EMCO Remote Console for Windows, schtask among others. The main advantage of these remote execution methods is that they are generic and not intrusive. However, on the downside, some of these methods have serious vulnerabilities, it is not possible to set priorities for the execution of the delegated commands, and some of these methods require prior configuration on the target machines. Our framework is able to handle both agent-based and agent-less implementations of the options. Naturally, it is possible to use an agent for some targets while other targets are hardened without the use of an agent in order to achieve the best possible performance.

\subsection{Implementation}

In our example implementation of the framework, we have made use of the framework's flexibility to utilize different mechanisms to interact with targets and implemented three different means of communication: a dedicated agent and two remote code execution mechanisms, SSH and PowerShell. These mechanisms have been chosen after performing extensive evaluation and testing. We found that, on the positive side, $\mathrm{SSH}$ is easy 
to use, OS-independent with scripting possibilities, and permits generic commands. On the negative side, it requires the installation of an $\mathrm{SSH}$ server and appropriate (and often distinct) configuration on different target platforms. The advantage of PowerShell is that it is installed by default on recent Windows operating systems and supports secure encrypted authentication and communication with scripting capabilities. The main drawback is that it is limited to machines running Windows. In addition, some configuration is needed. Other methods have severe security issues, e.g., PsExec transmits user credentials in clear text, or other shortcomings, for instance WMIC cannot retrieve the output after the execution of any command, i.e., it is not suitable for auditing.

Even though PowerShell is only available on the Windows platform, which entails that it is only an option for the alpha machines and targets running Windows, we still considered it in our analysis as Windows is widely used in industrial control. Note that the use of PowerShell on some machines does not imply that all machines must run Windows as several alpha machines with different characteristics and responsible for different groups of targets may be active at the same time.

Our current implementation can work with essentially all Windows regedit keys and secedit file settings, and all UNIX commands. Therefore, it is powerful enough to audit and enforce virtually any configuration on heterogeneous targets. In the following, an example implementation of an option is discussed briefly. A possible option for the security setting "firewall activated" with the associated value true for Windows machines would contain the following audit command:

$$
\text { netsh firewall show opmode }
$$

Netsh is a command-line scripting utility for the network configuration of Windows systems. The corresponding hardening command, again using Netsh, is:

\section{netsh firewall set opmode Enable}

As mentioned before, different baselines containing different options are required if there are multiple platforms. The option for the setting "firewall activated" for Linux systems could use, e.g., the Uncomplicated Firewall (ufw) to perform the auditing and hardening. The audit 1) and hardening 2) commands would be:

$$
\begin{aligned}
& \text { 1. ufw status } \\
& \text { 2. ufw --force enable }
\end{aligned}
$$

The methods to parse the output and compare the values are based on regular expressions for all options in our implementation. Since all data sent

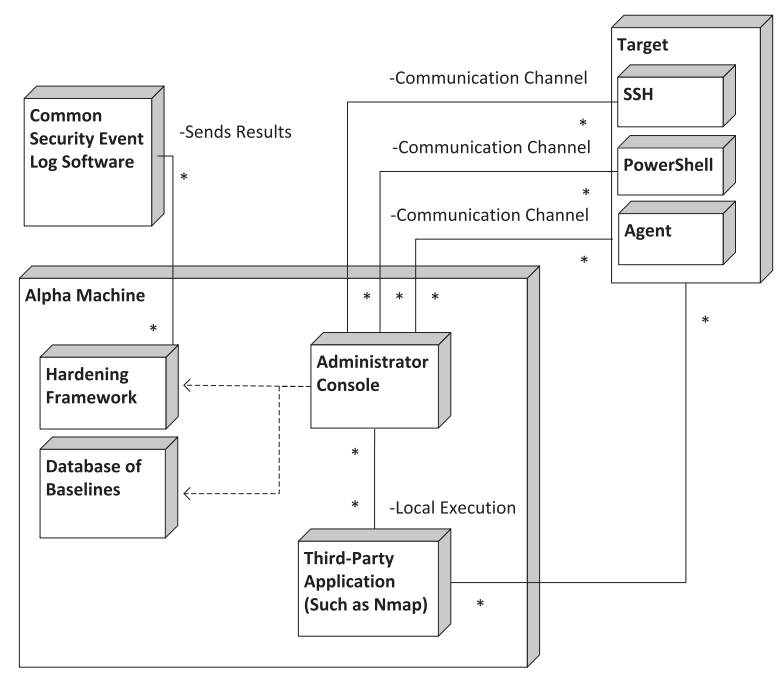

Figure 1: Components of the hardening framework.

back and forth between the alpha machines and the targets is XML text, regular expressions can be used to extract the desired values efficiently.

The diagram in Figure 1 depicts the possible components in the framework as well as a representation of how these elements are connected and interact. The Administrator Console, which uses the Hardening Framework's auditing and hardening functionality, is the main interaction component as it communicates with all targets and also with thirdparty applications, e.g., Nmap, a utility for network discovery and security auditing. ${ }^{3}$ The Administrator Console may call Nmap or other specialized security tools to extract information from the targets. In our implementation, port scanning is performed using Nmap. As discussed, there can be different communication channels between the Administrator Console and the targets; an agent and support for $\mathrm{SSH}$ and PowerShell are part of our implementation. The Administrator Console obtains the security policies from a database of baselines where the policies are maintained. In addition, the framework can be integrated with other security event logging tools to further analyze the current system status or to simply report audit and hardening results for visualization or archiving.

\section{EVALUATION}

Since security policies vary significantly from system to system, it is generally not feasible to measure the attained degree of protection. In addition, the framework's main purpose is to enforce existing security policies autonomously, rather than to generate or adapt them. Thus, in order to be able to use the approach for a large system, the efficiency of the proposed framework is important. A low bandwidth and CPU consumption and a short

\footnotetext{
${ }^{3}$ See www.nmap.org
} 
completion time are essential to support (industrial) applications in large networks where the hardening must occur frequently.

We have therefore implemented and evaluated the performance of the proposed framework. In particular, we have analyzed the performance of the three different execution modes of our hardening system, i.e., agent-based, SSH, and PowerShell, and identified potential optimizations. Finally, we have also evaluated the scalability of the approach.

\subsection{Setup}

In order to test our implementation of the framework, we ran it on up to 500 Windows 2008 and Linux servers in Amazon's Elastic Computing Cloud. This cloud platform was chosen because its bandwidth and latency characteristics are much closer to industrial control networks compared to, e.g., PlanetLab ${ }^{4}$, and also because it allows us to easily increase the number of machines used in the experiments, a prerequisite for scalability testing.

In our setup, we used a single active alpha machine running on an i7 2.2Ghz PC with 8GB of RAM and the targets ran on low-end machines with $613 \mathrm{MB}$ of memory. It is important to note that the performance of SSH varies significantly between Windows and Linux. Therefore, all experiments involving PowerShell are restricted to targets running Windows in order to get a fair comparison. The difference to SSH on Linux will be discussed later.

We have measured the execution time of a hardening operation, which is defined as the time between the sending of the command/baseline and the completion time of the operation. The CPU consumption is measured as an average CPU usage over one second and over the number of cores. The bandwidth consumption sums up the traffic volume between the alpha machine and the targets. Keeping all of these measures small enables the system to scale to a large number of targets.

\subsection{Comparison of Execution Methods}

In order to compare the hardening performance of different execution methods, we have used one Windows server 2008 running in the cloud and a baseline that contains a single option. Since an option is the smallest unit for auditing and hardening in our framework, testing a single option reveals the overhead of each method. Furthermore, the total load that our framework imposes on the system directly depends on the computational cost and bandwidth usage when executing the commands in the option and also the periodicity of the audits. Since the "right" periodicity depends on the characteristics of the considered system, it is an input parameter for our framework, i.e., the

\footnotetext{
${ }^{4}$ See www.planet-lab.org
}

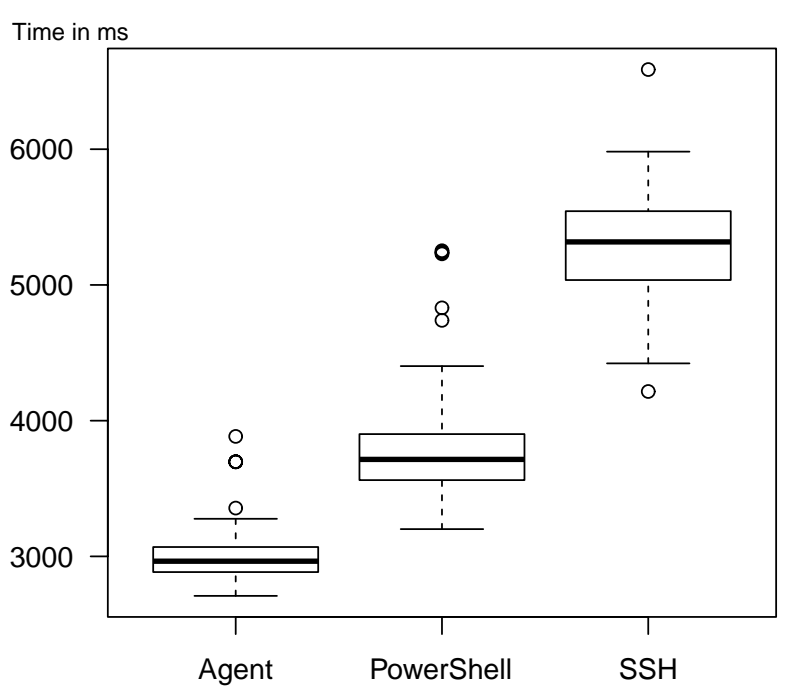

Figure 2: Command execution times in milliseconds for each execution method.

framework currently does not determine the intervals between the periodic audits itself. The "firewall activated" option outlined in the previous section is used for this test.

The goal of the evaluation was to test a) the execution time, b) the CPU consumption on the target machine, and c) the total bandwidth consumption. The CPU consumption on the alpha machine for a single option is low (a few percentage points) independent of the communication method. The impact of having larger baselines and target networks is evaluated in Section 4.4.

\subsubsection{Execution Time}

Figure 2 visualizes the execution times over 200 runs when auditing one setting for each of the three hardening methods. On average, the agent is faster than PowerShell, which in turn is faster than $\mathrm{SSH}$. As expected, the variance is also minimized when using the agent.

The exact measurement results are summarized in the following table:

\begin{tabular}{|c|c|c|c|}
\hline & Agent: & PowerShell: & SSH: \\
\hline Mean (ms): & 2981 & 3763 & 5288 \\
\hline Std. dev. (ms): & 161 & 320 & 344 \\
\hline
\end{tabular}

\subsubsection{CPU Consumption}

Figure 3 shows how the CPU consumptions over a time window of 1500 seconds is dispersed over time (from left to right). Hence, there are 1500 data points as each measurement is computed over one second. As the execution time is in the order of seconds as discussed in the previous section, the hardening is performed a few hundred times in this time interval. On average $3.1 \%$ of the total CPU capacity is used for the agent (standard deviation $\sigma=7.4 \%$ ), whereas PowerShell requires $7 \%(\sigma=12.4 \%)$. While the 

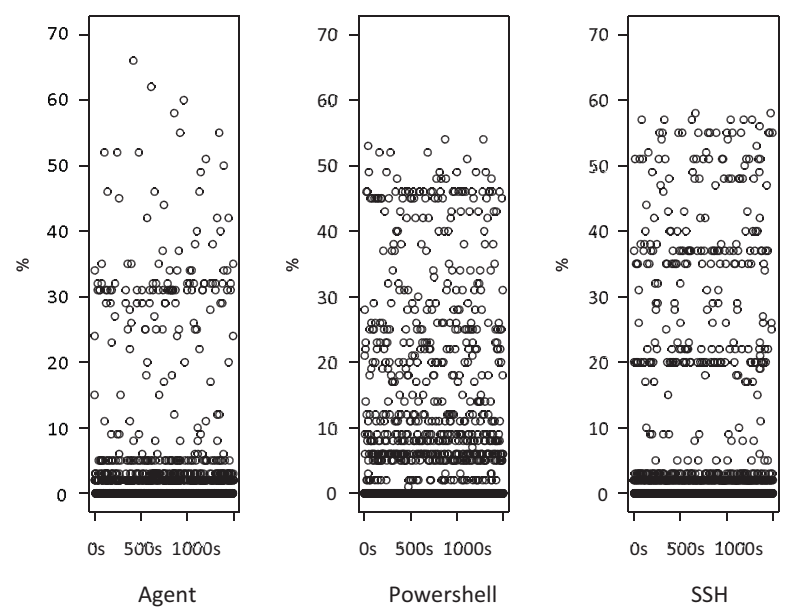

Figure 3: $C P U$ consumption of a target during auditing for each execution method.

average CPU consumption of SSH is slightly lower than PowerShell at $6.25 \%$, the variance is larger $(\sigma=13.5 \%)$ with small concentrations at 20 and $38 \%$. The agent not only consumes less CPU power, it can further bound the CPU consumption by setting a priority for the execution of commands, which shows that it is superior when bounding the CPU consumption is crucial.

\subsubsection{Bandwidth Consumption}

The bandwidth consumption has been measured by capturing the transmitted packets using Wireshark, a widely used network protocol analyzer. ${ }^{5}$ Figure 4 shows the network traffic between the alpha machine and a single target over a period of 100 seconds when using the agent, PowerShell, and SSH. Figure 4(a) reveals that the data rate is quite small when using the agent, with peaks of around $5 \mathrm{~KB} / \mathrm{s}$ over short periods of time. Figure 4(b) shows that the bandwidth consumption is significantly larger when using PowerShell, reaching approximately $25 \mathrm{~KB} / \mathrm{s}$. Finally, as shown in Figure 4(c), SSH requires even less bandwidth than the agent peaking at $2.5 \mathrm{~KB} / \mathrm{s}$. However, SSH needs slightly more time to exchange the same data.

As these figures reveal, the throughput for all three methods is quite regular, i.e., there are no sudden and unexpected peaks. Our evaluation shows that the agent exhibits the best performance on average in terms of execution time and CPU performance, while SSH requires the smallest amount of bandwidth. For networks with strong bandwidth constraints or devices with low CPU power, PowerShell is not recommended. In this scenario, using both an agent and an agent-less method, e.g. SSH, should be considered.

\footnotetext{
${ }^{5}$ See www.wireshark.org
}



(a) Agent.



(b) PowerShell.

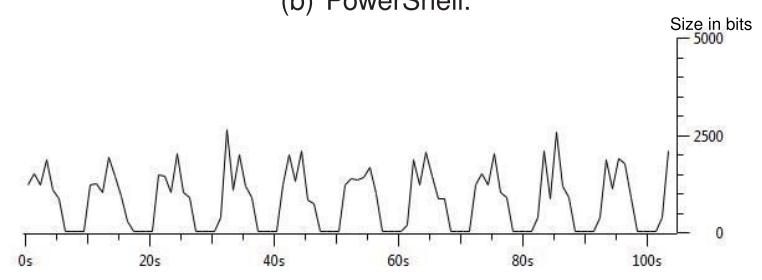

(c) $\mathrm{SSH}$.

Figure 4: Bandwidth consumption between the alpha machine and a target for 100 seconds of communication using the agent (a), PowerShell (b), and SSH (c).

\subsection{Baseline Performance}

Since a real-world security policy typically contains several security settings, we proceed by measuring the performance of our implementation when applying more comprehensive baselines with multiple hardening settings. In particular, we defined a oneoption baseline (B1), a five-option baseline (B5), a ten-option baseline (B10), and an optimized tenoption baseline $\left(\mathrm{B} 10^{*}\right)$. Baseline $\mathrm{B} 1$ contains the same option as in the previous experiment, whereas the other baselines contain additional options. For example, B5 additionally contains the options "minimum password length", "maximum password age", "no guest account", "USB disabled". Note that the type of commands in the baselines is similar and therefore the execution time of each command is approximately the same, which allows us to determine the effect of increasing the size of the baselines. These specific options were chosen since there are commands on both Windows and Linux to audit and harden them. For the baselines B5, B10, and $\mathrm{B} 10^{*}$, the commands are bundled to reduce the time required to transfer them and thereby minimize the bandwidth consumption. The baseline $\mathrm{B}^{10^{*}}$ is optimized in the sense that the results of five options can be re-used to compute the results for the remaining five options using semantic analysis. For example, one option determines the open ports. This 




Figure 5: Command execution times in milliseconds for each baseline using the agent.

information is used to deduce whether certain services using specific ports are accessible. As this is a common scenario in practice, it is worth examining the benefit of analyzing the overlap between the auditing results.

\subsubsection{Execution Times}

The execution times of the four baselines using the agent are shown in Figure 5, which clearly shows the advantage of bundling the commands: executing baseline B10 (B5) takes roughly $4 \mathrm{~s}$ (3.5s), whereas executing the single-option baseline B1 already takes $3 \mathrm{~s}$ on average. Moreover, the figure further showcases the positive effect of semantic optimization. Baseline B10* only needs a little more time to completion compared to baseline B5 as it merges the ten commands for the individual options into five slightly more complex commands.

The results for PowerShell are essentially the same as the results for the agent but all execution times are marginally larger. We measure even larger execution times for SSH, which is consistent with Figure 2. As mentioned before, the result is quite different on Linux targets. Figure 6 shows that SSH on Linux achieves much lower execution times: The execution time of $\mathrm{B} 10^{*}$ is only a few milliseconds larger than the execution time of B1. This result shows that the implementation and configuration of the SSH server can have a substantial impact on the hardening performance.

\subsubsection{CPU Consumption}

The CPU consumption of the agent-based method grows from $3.1 \%$ on average $(\sigma=7.4 \%)$ for B1, as discussed in Section 4.2.1, to $4.8 \%$ for both B5 and B $10^{*}(\sigma=10.3 \%)$, whereas the consumption for B10 is around $9.1 \%(\sigma=19.5)$. This shows that bundling multiple commands into a single baseline results in a slow increase in CPU consumption. Moreover, it is

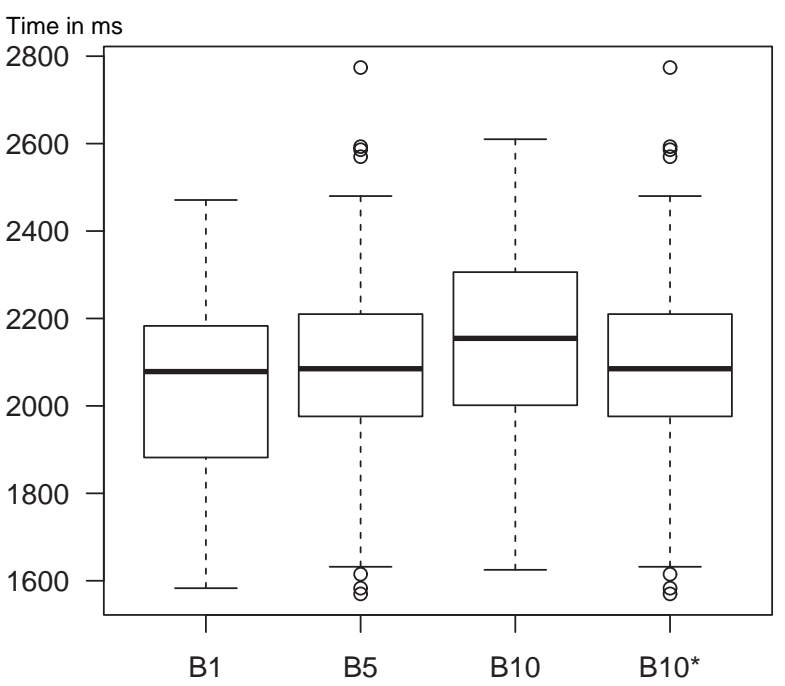

Figure 6: Command execution times in milliseconds for each baseline using SSH on Linux targets.

evident that semantic optimization pays off as B10* almost outperforms B10 by a factor of 2 .

While the averages and standard deviations for PowerShell are slightly larger, each by roughly 4 to 5 percentage points, the same conclusions can be drawn. Due to space limitation and the fact that the CPU consumption heavily depends on the configuration of the SSH server as mentioned before, we omit the discussion of the consumption for SSH.

\subsection{Scalability}

We evaluated the scalability of our solution by increasing the number of machines to harden. For this purpose, we set up 100 Windows server 2008 machines (targets) running in the EC2 cloud and used the same one-option baseline again. The performance of the active alpha machine is tested under increasing load to verify that our framework is capable of auditing and hardening large networks.

\subsubsection{Execution Times}

The execution times for the audit are shown in Figure 7 . The $x$-axis represents the number of concurrently executed threads, while the $y$-axis represents the total time required to harden the 100 targets. When the number of threads increases, a limit of 15 seconds for both PowerShell and SSH is reached using 30 threads. Figure 7 again reveals the benefit of having a custom-tailored agent, achieving the lowest execution times when using roughly 50 threads.

This experiment further shows the scalability of the approach, which can further be improved by distributing the workload among multiple active alpha machines. 


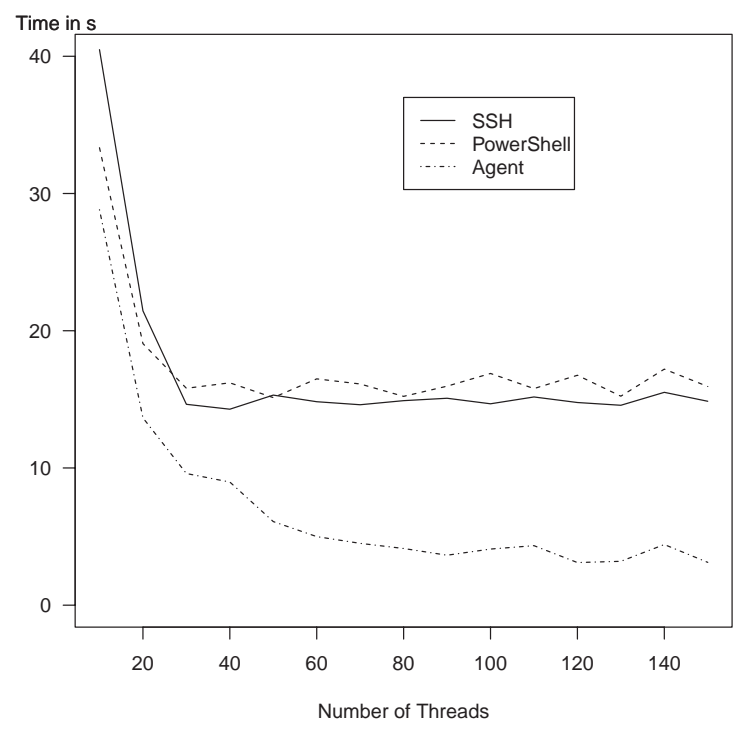

Figure 7: Audit time in seconds for 100 computers for an increasing number of threads.

\subsubsection{CPU Consumption}

The CPU consumption for a varying number of threads is shown in Figure 8. 100\% CPU consumption is reached with roughly 50 threads when using PowerShell, while the agent requires the least CPU power, staying below $50 \%$ even with 140 threads.

\subsubsection{Scalability}

In order to test the overall scalability of the approach, we have increased the number of targets up to 500 and measured the overall execution time and the CPU consumption on the single alpha machine. Note that the scalability can be increased easily by adding more alpha machines. The number of threads has been increased simultaneously, i.e., a separate thread is started for each target machine. Figure 9 and 10 illustrate the results. While the $x$-axis represents the number of targets (which corresponds to the total number of threads), the $y$-axis in Figure 9 represents the total required time to harden the targets. Using the PowerShell method, we can observe an almost linear growth, which can be explained by the overhead when executing PowerShell scripts. For SSH, the same argumentation holds, while the agent based solution outperforms the other solutions and requires an almost constant amount of time. This result shows that the agent is able to execute its tasks more efficiently than a multi-purpose tool, thereby reducing the load on the alpha machine significantly. Figure 10 reveals that PowerShell quickly uses up the entire CPU, whereas the CPU consumption for SSH grows linearly, reaching around $90 \%$ for approximately 100 targets. The CPU consumption also grows linearly when agents are used; however, the consumption grows slowly, reaching roughly $50 \%$ with 500 targets.

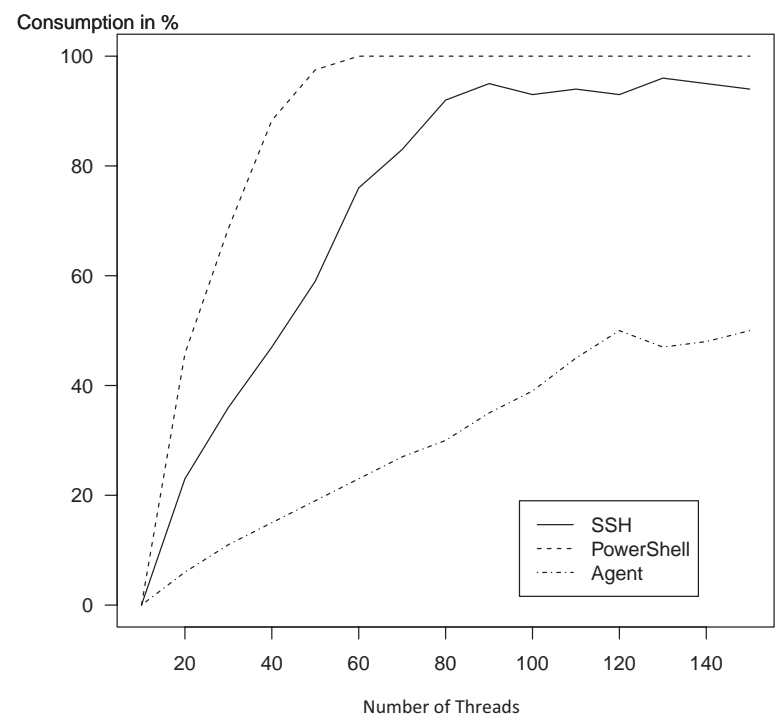

Figure 8: CPU consumption percentage using 100 targets for an increasing number of threads.

In summary, we have seen that with an increasing number of target machines (up to 500 machines running in the cloud), the agent-based hardening method is superior to SSH and PowerShell. As the agent generally performed well in most experiments, the agent-based approach can be considered the method of choice. If installing additional software is not an option (due to regulations or other reasons), then an agent-less, in particular $\mathrm{SSH}$ based, approach also works reasonably well. On the Linux platform, we found that issuing commands over SSH directly may even outperform the agentbased approach.

\section{CONCLUSION}

Automated hardening is a crucial step towards ensuring a high level of security particularly in dynamic control environments. We have presented a framework allowing a networked system of heterogeneous devices to periodically harden itself autonomously, which is beneficial for industrial and enterprise applications. The evaluation of our implementation using up to 500 machines on the EC2 platform shows that the framework is efficient and scalable and therefore suitable for large industrial networks.

In addition, the evaluation analyzes and compares three different communication methods, $\mathrm{SSH}$, PowerShell, and a dedicated agent, to maintain secure configurations. In most of the measurements, the agent-based approach yields the best results, whereas SSH is generally the appropriate choice among the agent-less methods as a suitable alternative whenever agents are considered too invasive.

A direction for future research is to extend the experiments to take a larger variety of operating 


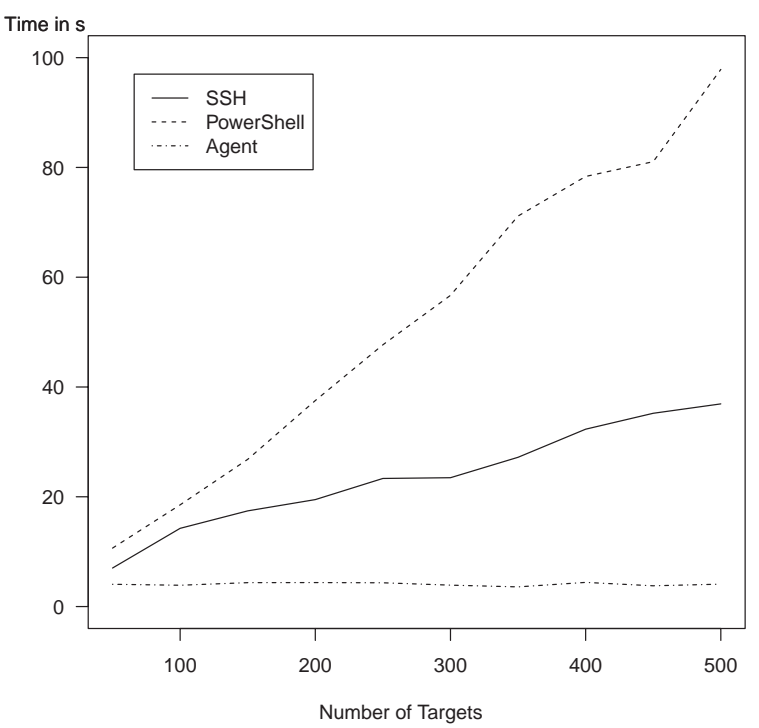

Figure 9: Execution time in seconds for an increasing number of targets/threads.

systems into account, including (real-time) operating systems such as VxWorks ${ }^{6}$, which are commonly used in the ICS domain. It would be interesting to study the feasibility of periodically running audits and hardening steps, with and without agents, on machines with real-time constraints and little computational reserves. The development of new techniques may be required when extending the scope to include such constraint environments.

\section{REFERENCES}

Balasubramaniyan, J. S. et al. (1998) An architecture for intrusion detection using autonomous agents. In: Proc. 14th Annual Computer Security Applications Conference. 13-24.

Burgess, M. (1998) Computer immunology. In: Proc. 12th USENIX Large Installation System Administration Conference (LISA), 283-298.

Forrest, S., Hofmeyr, S. A., and Somayaji, A. (1997) Computer immunology. Commun. ACM, 40 (10). 88-96.

Goldsack, P. et al. (2003) SmartFrog: Configuration and automatic ignition of distributed applications. In: HP Openview University Association Conference.

Helmer, G. et al. (1998) Intelligent agents for intrusion detection. In: Information Technology Conference, 121-124.

Hochberg, J. et al. (1993) NADIR: An automated system for detecting network intrusion and misuse. Comput. Security, 12 (3). 235-248.

\footnotetext{
${ }^{6}$ See www.windriver.com/products/vxworks
}

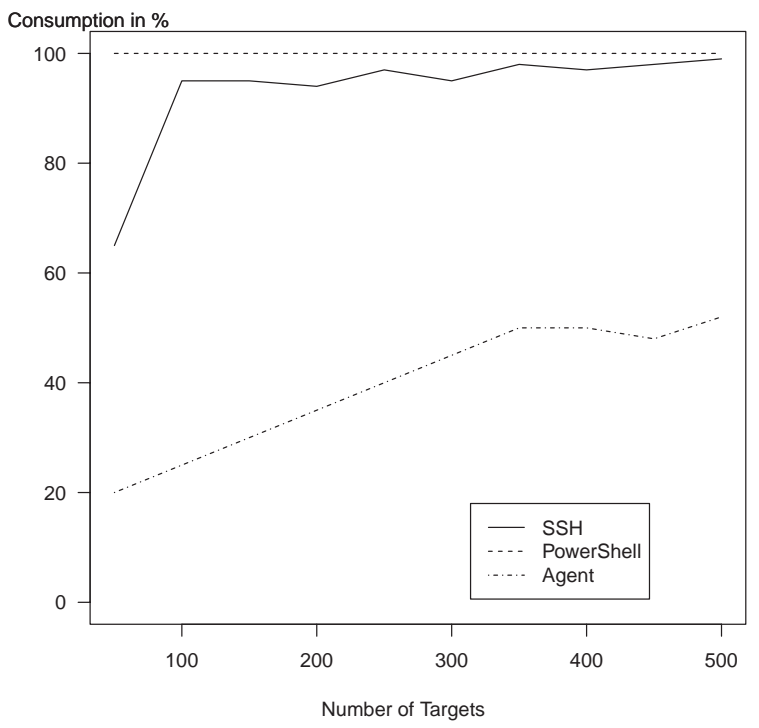

Figure 10: CPU consumption for an increasing number of targets/threads.

Jabbour, G. G. and Menasee, D. A. (2008) Policy-based enforcement of database security configuration through autonomic capabilities. In: 4th International Conference on Autonomic and Autonomous Systems (ICAS), 188-197.

Karjoth, G. (2003) Access control with IBM Tivoli access manager. ACM Trans. Inform. Syst. Security (TISSEC), 6 (2). 232-257.

Lamport, L. (1998) The part-time parliament. ACM Trans. Comput. Syst., 16 (2). 133-169.

Menascé, D. A. and Bennani, M. N. (2003) On the use of performance models to design selfmanaging computer systems. In: Proc. Computer Measurement Group Conference (CMG), 7-12.

Mukherjee, B., Heberlein, L. T., and Levitt, K. N. (1994) Network intrusion detection. IEEE Netw., 8 (3). 26-41.

Porras, P. A. and Neumann, P. G. (1997) EMERALD: Event monitoring enabling response to anomalous live disturbances. In: Proceedings of the 20th National Information Systems Security Conference. 353-365.

Spafford, E. H. and Zamboni, D. (2000) Intrusion detection using autonomous agents. Comput. Netw., 34 (4). 547-570.

Yemini, Y., Konstantinou, A. V., and Florissi, D. (2000) NESTOR: An architecture for network self-management and organization. IEEE J. Sel. Areas Commun., 18 (5), 758-766. 